\title{
Photospheric and coronal abundances of solar-type stars with planets: the case of $\tau$ Bootis
}

\author{
Antonio Maggio ${ }^{1}$, Jorge Sanz-Forcada ${ }^{2}$ and Luigi Scelsi ${ }^{1}$ \\ ${ }^{1}$ INAF - Osservatorio Astronomico di Palermo, \\ piazza del Parlamento 1, I-90134, Palermo, Italy \\ email: maggio@astropa.inaf.it \\ ${ }^{2}$ Centro de Astrobiologia, CSIC-INTA, European Space Astronomy Centre, \\ Apartado 78, E-28691 Villanueva de la Canada (Madrid), Spain \\ email: jsanz@laeff.inta.es
}

\begin{abstract}
Bootis, a middle-aged solar analogue, well known for the presence of a close-in Jovian mass planet. We employ the results of this study, based on high-resolution optical and X-ray spectra, to address the issue of abundance stratification vs. First Ionization Potential (FIP) in the outer stellar atmospheres of solar-type stars with and without planets.
\end{abstract}

Keywords. stars: abundances, stars: activity, stars: coronae, stars: late-type; X-rays: stars

\section{Introduction}

Chemical abundances in solar-type stars still represent an open issue, in many respects: the photospheric abundance of elements with high first ionization potentials, like Argon and Neon, is poorly known; coronal and photospheric abundances appear to be different in many cases, with a possible dependence on "unconventional" parameters like the stellar activity level; photospheres of planet-hosting stars are known to be metal-rich, but does this peculiarity apply also to the chemical composition of the outer stellar atmosphere?
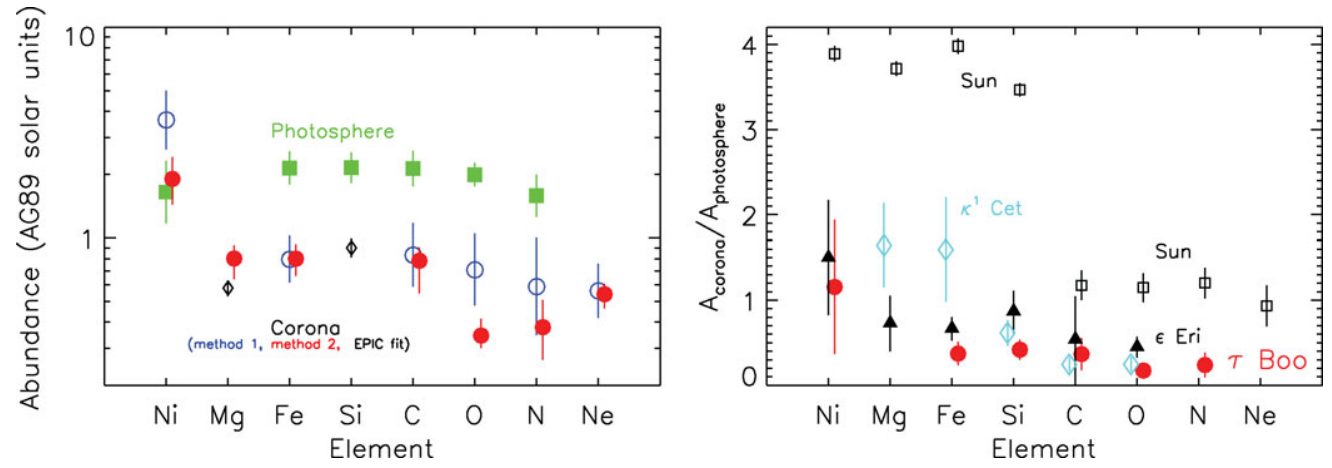

Figure 1. Left panel: Photospheric and coronal abundances of $\tau$ Boo for individual elements, sorted by increasing FIP, relative to the solar photospheric composition (Anders \& Grevesse 1989). Right panel: Comparison of coronal to photospheric abundance ratios of $\mathrm{F}-\mathrm{G}$ dwarfs with different activity levels. 


\section{Observation and analysis results}

Tau Bootis (HD 120136) is a $1.3 M_{\odot}$ F7V star, located at $15.6 \mathrm{pc}$ from the Sun. It harbors a planet with a mass $M \sin i \approx 3.9 M_{\mathrm{J}}$, discovered by Butler et al. (1997) in a 3.31 day period, synchronized with the stellar rotation period. Element abundances in its photosphere (Gonzalez \& Laws 2007, Fig. 1 left panel) are systematically higher by a factor $\approx 2$ with respect to the solar ones.

Tau Bootis was observed in June 2003 for $\approx 50$ ksec. Its X-ray light curve shows just some low-level variability, typical of the quiescent coronal emission of solar-type stars.

Coronal abundances were determined simultaneously with the plasma emission measure distribution from the analysis of RGS and EPIC spectra. We employed two different and independent methods (Sanz-Forcada et al. 2003, Scelsi et al. 2004) for a detailed line-based analysis of the RGS spectra, with the aim to look for systematic uncertainties. EPIC spectra were also employed to constrain the amount of high-temperature plasma and the abundances of a few more elements without spectral signatures in RGS spectra.

The results obtained with the two methods are in good agreement (Fig. 1, left panel), and clearly indicate that the coronal abundances of $\tau$ Boo are significantly lower than photospheric ones.

\section{Conclusions}

Tau Bootis is a coronal X-ray source with a level of magnetic activity intermediate between that of the quiet Sun (Peres et al. 2000) and those of younger F-G stars. In Fig. 1 (right panel) we compare the coronal to photospheric abundance ratios of $\tau$ Boo (method 2) with those of the Sun (Feldman \& Laming 2000) and two other intermediateactivity G-type stars: $\epsilon$ Eri (Sanz-Forcada et al. 2004), another planet-hosting star, and $\kappa^{1}$ Cet (Telleschi et al. 2005) without any known planet.

The pattern of coronal to photospheric abundance ratios vs. FIP for $\tau$ Boo is almost flat (no FIP bias, with possible exception for $\mathrm{Ni}$ ), and suggests that its coronal characteristics are similar to those of other intermediate-activity stars with planets, like $\epsilon$ Eri.

\section{References}

Anders, E. \& Grevesse, N. 1989, Geochim. Cosmochim. Acta, 53, 197

Butler, R. P., Marcy, G. W., Williams, E., Hauser, H., \& Shirts, P. 1997, ApJ, 474, L115

Feldman, U. \& Laming, J.M. 2000, Physica Scripta, 61, 222

Gonzalez, G. \& Laws, C. 2007, MNRAS, 378, 1141

Peres G., Orlando S., Reale F., Rosner R., \& Hudson H. 2000, ApJ, 528, 537

Sanz-Forcada, J., Brickhouse, N. S., \& Dupree, A. K. 2003, ApJS, 145, 147

Sanz-Forcada, J., Favata, F., \& Micela, G. 2004, A\& $A$, 416, 281

Scelsi, L., Maggio, A., Peres, G., \& Gondoin, P. 2004, A\&A, 413, 643

Telleschi, A., Güdel, M., Briggs, K., Audard, M., Ness, J.-U., \& Skinner, S. 2005, A\&A, 622, 653 